\title{
Desarrollo de Habilidades Básicas de Pensamiento Crítico en el Contexto de la Enseñanza de la Física Universitaria.
}

\author{
Inmaculada Aznar ${ }^{(1)}$ e Ignacio Laiton ${ }^{(2) *}$ \\ (1) Universidad de Granada, Facultad de Ciencias de la Educación. España. \\ (2) Escuela Tecnológica Instituto Técnico Central, Calle 13 \# 16-74, Bogotá, Colombia. \\ (ilaiton@itc.edu.co; iaznar@ugr.es) \\ *Autor a quien debe ser dirigida la correspondencia
}

Recibido May. 20, 2016; Aceptado Jul. 22, 2016; Versión final Ago. 25, 2016, Publicado Feb. 2017

\begin{abstract}
Resumen:
El presente artículo muestra los resultados de una investigación orientada a generar y evaluar una propuesta pedagógica que incida positivamente en el nivel de habilidades de pensador crítico de los estudiantes universitarios desde el área de física. Se detectó previamente la ausencia de este tipo de habilidades en los estudiantes de primeros semestres de ingeniería por lo que se propuso generar estrategias pedagógicas que aporten a la solución del asunto. Se diseña una intervención pedagógica basada en teorías sobre enseñanza de habilidades de pensamiento. Se aplica al curso designado como muestra, teniendo un grupo control, y se selecciona la forma más adecuada para medir el nivel de habilidades de pensamiento crítico. Se aplicaron los test al comienzo y al final de la intervención pedagógica, procesando los datos estadísticamente. Los resultados muestran variaciones significativas positivas en algunos de los aspectos evaluados.
\end{abstract}

Palabras clave: educación; pensamiento crítico; habilidades; solución de problemas; intervención pedagógica

\section{Development of Basic Skills Critical Thinking in the Context of Teaching of University Physics}

\begin{abstract}
:
This article shows the results of an investigation oriented to generate and evaluate a pedagogical proposal that positively affects the level of skills as critical thinkers of college students from the area of physics. Detected the absence of such skills in students of first semesters of engineering, it was proposed to generate teaching strategies that contribute to the solution of this matter. An educational intervention based on theories of teaching thinking skills was designed. This was applied to a course designated as the experimental sample, having also a control group, and the most appropriate way for measuring the level of critical thinking skills was selected. Tests at the beginning and end of the educational intervention were applied, the data was statistically processed. The results show significant positive changes in some of the aspects evaluated.
\end{abstract}

Keywords: education; critical thinking; thinking; abilities; solve problems; educational intervention 


\section{INTRODUCCIÓN}

El trabajo investigativo actual se asume desde la acción de generar y evaluar una estrategia pedagógica que se intuya enriquezca y promueva las habilidades de pensamiento crítico del estudiante desde el campo disciplinar de la física. Se prevé que el desarrollo de habilidades de pensador crítico implica una educación integral, que desarrolle sus competencias y su característica fundamental de ser en el sentido de saber movilizar los conocimientos que se poseen en las diferentes y cambiantes situaciones que se presentan en la práctica (Aznar et al, 2011). Se debió probar y errar, intentar y fracasar, con el objetivo de filtrar información sobre las características globales o generales de una estrategia pedagógica exitosa en tal sentido, se parte de la hipótesis de que sí es posible generar una intervención pedagógica planeada y organizada, enfocada a la generación y desarrollo de habilidades de pensamiento crítico desde el campo disciplinar de la física, que se refleje en la evolución tales habilidades en los estudiantes. En los siguientes apartados se propone el sustento teórico del proyecto desde los elementos no disyuntos de la didáctica de las ciencias, la solución de problemas y la enseñanza y evaluación de las habilidades de pensamiento crítico. Se muestran también la metodología aplicada desde el punto de vista investigativo, del diseño y aplicación de la intervención pedagógica, así como desde su evaluación. Finalmente se muestran los resultados develados a través del tratamiento estadístico de los datos, encontrando luces importantes para trabajos posteriores.

En busca de los antecedentes del problema se encuentra en el ámbito de la enseñanza de pensamiento crítico e intervenciones didácticas en esta línea, en educación superior, encontramos trabajos como el desarrollado por Castiblanco y Vizcaino (2006), quienes relacionan en su título la enseñanza de la física con el desarrollo de pensamiento crítico, enfocado desde la perspectiva de proyectos de aula tales como el globo aerostático, la catapulta y otros. Por otro lado, Girelli, Dima, Reinoso y Baumann (2010) realizan un estudio sobre el desarrollo de habilidades de pensamiento crítico y superior en dos estudiantes de física en educación superior a través de las opiniones de sus docentes, no de una medición directa.

También Lara-Barragán y Cerpa (2014) desarrollan un trabajo con el objetivo de desarrollar habilidades de pensador crítico en los estudiantes de una universidad en Guadalajara, México, sin embargo, su experiencia se centra en el paradigma constructivista a la hora del diseño de la intervención didáctica, nuevamente difiere en aspectos importantes como el modo de evaluación del pensamiento crítico y en su enfoque didáctico principal. Otros trabajos relacionados como los de Franco, Almeida y Saíz (2014), Beltrán y Torres (2009), Olivares y Heredia (2012), Osman et al. (2015), y Betancourth (2015), insisten en la importancia de la enseñanza del pensamiento crítico en las disciplinas. Se destaca eso sí la importancia que en diferentes sectores y países ha surgido alrededor del tema de la enseñanza del pensamiento crítico. Se justifica proponer ideas y ejecutar proyectos que sirvan para evaluar en la práctica cotidiana de las universidades Latinoamericanas si este tipo de enseñanza puede ser efectivo a mediano y largo plazo.

En el campo de la enseñanza de las ciencias y la solución de problemas, son numerosos los aportes que se encuentran en la literatura, Gangoso (1999) presenta un importante compendio de una gran parte de los trabajos desarrollados hasta esta fecha en el campo de la resolución de problemas, que además involucran de manera directa la enseñanza de las ciencias, en algunos casos la física en particular, área de desarrollo del actual trabajo, se destacan de manera importante la clasificación que la autora hace que nos condujeron a varios trabajos enfocados en analizar el contenido del problema, enumerando aspectos como la estructura, la sintaxis, el enfoque, etc. Continuando con una nueva búsqueda encontramos trabajos más recientes como el Benegas y Villegas (2011), e Inzunza y Brincones (2010) en la Universidad de Alcalá quienes resaltan los procesos mentales seguidos por los estudiantes y aspectos como la importancia del enunciado en el contexto de la resolución de un problema en física, diferenciando los enunciados de los ejercicios de final de capítulo, versus enunciados más relacionados con la vida cotidiana.

Se resume que no se encontraron trabajos tendientes a gestar estrategias pedagógicas en un área disciplinar como la física, que tiendan a generar la aparición o mejoramiento de las habilidades de pensamiento crítico en los estudiantes, motivando su aprendizaje activo, teniendo en cuenta elementos cognitivos y de pensamiento, que además sean evaluados mediante test estandarizados de pensamiento crítico como el trabajo actual.

El aspecto central del presente trabajo lo constituye la posibilidad de la enseñanza o la generación de habilidades de pensador crítico en los estudiantes de física mecánica de la Escuela Tecnológica Instituto Técnico Central ETITC de Bogotá. La generación de una estrategia pedagógica que facilite tal objetivo implica una fundamentación importante en el tema del pensamiento crítico, su naturaleza, su enseñanza y su evaluación. La intervención pedagógica se estructura sobre los fundamentos teóricos aportados por Dewey (2007), Perkins (2008), Ennis (2000), entre otros. El término pensamiento crítico ha sido usado desde siempre en las descripciones pedagógicas sin que exista una precisión absoluta en cuanto a su 
significado concreto, algunos autores lo equiparan al pensamiento creativo (Dewey, 2007), otros hablan de pensamiento superior, mientras que Mayer (2002) lo equipara con la solución de problemas. Sin embargo en lo que coinciden la mayoría de los autores es en ciertas características propias de un pensador crítico tales como; análisis de argumentos, evaluación de la calidad de una fuente, formulación y valoración de juicios de valor, solución de problemas, toma de posición frente a un tema, etc. Se trata de generar individuos que aprendan a aprender, razonen, reflexionen, que no sean simplemente receptores de conocimiento, sino más bien generadores de ideas a partir de él, que sean capaces de tomar decisiones y adoptar posturas tendientes a fundamentar sus creencias y argumentar sus actuaciones.

La enseñanza del pensamiento crítico varía básicamente entre quienes defienden que puede ser enseñado como área individual, independiente de las asignaturas propias del currículo escolar como el profesor Edward De Bono, y el profesor Reuven Feuerstein (Maclure y Davies, 2003), y quienes defienden que las habilidades de pensamiento se enseñen a través de las áreas disciplinares. Se trata de diseñar estrategias que transformen radicalmente la enseñanza de las asignaturas de cualquier currículo escolar (Maclure y Davies, 2003), centrándose no en los contenidos como habitualmente lo hacen, sino más bien tomarlo como caballo de batalla sobre el cual se enseñe a pensar y se fortalezcan en general las facultades intelectuales de los estudiantes.

Boisvert (2004) afirma y conduce la forma en que es posible estructurar una propuesta para el aula de clase, coherente y productiva que promueva el desarrollo del pensamiento crítico en la enseñanza de las áreas disciplinares. Se estructura la intervención pedagógica a través de esta propuesta, realizando en primer lugar un acercamiento profundo al tema de la naturaleza del pensamiento crítico desde los diferentes autores reconocidos en el tema, dentro de los que se pueden citar al propio Ennis (2000), Perkins (2008), Lipman (2001), Browne y Keeley (2015), posteriormente se ponderan las habilidades de pensamiento crítico posibles de imbuir en un curso disciplinar, dentro de las que se destacan; evaluación de la credibilidad de una fuente, uso del proceso de solución de problemas, pensamiento deductivo, pensamiento inductivo, razonamiento, toma de decisiones. Finalmente, el aspecto evaluativo de la intervención que debe ser coherente y adecuada al diseño elegido (Boisvert, 2004)

En segundo lugar, se aborda el tema de la resolución de problemas, dada su importancia en el desarrollo del pensamiento, que se ha enunciado desde diferentes perspectivas tanto en la enseñanza en general como en la enseñanza de las ciencias, en particular en la física. Pozo (1999) afirma que la solución de problemas fomenta en el estudiante la capacidad de aprender a aprender. También afirma que tal enseñanza puede dotar a los estudiantes de destrezas eficaces, así como la creación de hábitos tendientes a buscar siempre soluciones a situaciones en cualquier contexto. Mayer (2002), Polya (1965) o Boisvert (2004), resaltan la importancia de una enseñanza centrada en la resolución de problemas, argumentando que proporciona a los alumnos destrezas y estrategias que lo habilitan para generar hábitos de razonamiento sistemático y riguroso que además tienen la posibilidad de ser aplicados en situaciones de la vida cotidiana del individuo. Interesa aquí el hecho de que parte fundamental de la estrategia pedagógica aplicada se basa en el diseño, aplicación y solución de problemas analíticos en física, claramente problemas mal definidos pero que exigen más elementos cognitivos por parte del estudiante.

\section{METODOLOGÍA}

Se siguió una metodología cuantitativa, con un diseño experimental, entendiéndose que es uno de los métodos más poderosos de investigación cuando se pretende identificar relaciones causales entre las variables (Colás et a., 2009), como en el actual caso, donde se pretende medir la incidencia de la variable independiente: estrategia pedagógica basada en la enseñanza del pensamiento crítico, sobre la variable dependiente: existencia de habilidades de pensamiento crítico en los estudiantes. Dentro de los diseños experimentales, se eligió el denominado diseño pretest - postest con grupo control.

\begin{tabular}{|cccc|}
$\mathrm{R}$ & $\mathrm{O}_{1}$ & $\mathrm{X}$ & $\mathrm{O}_{2}$ \\
$\mathrm{R}$ & $\mathrm{O}_{3}$ & & $\mathrm{O}_{4}$ \\
\hline
\end{tabular}

Fig. 1: Diseño de la Intervención Pedagógica

En la figura 1 se observa la presencia de los dos grupos $(R)$, en ambos la variable dependiente es medida antes $\left(\mathrm{O}_{1}\right.$ y $\left.\mathrm{O}_{3}\right)$ y después $\left(\mathrm{O}_{2}\right.$ y $\left.\mathrm{O}_{4}\right)$ de la intervención $(\mathrm{X})$ sobre el primer grupo y el otro como grupo control. Para la secuencia de aplicación $\left(\mathrm{O}_{n}\right)$ se usó la prueba PENCRISAL, al comienzo y al final del semestre académico. Los grupos fueron asignados al azar, dado que son los cursos que regularmente dicta el docente investigador. Las variables intervinientes identificadas son la edad y el estrato social del que 
provienen los estudiantes de la muestra, variables que se determinó no son influyentes dado que tanto el rango de edades de los dos grupos, el intervenido y el de control son en promedio iguales, 19.66 y 19.46 respectivamente, mientras que el estrato social al que pertenecen no presentan diferencias estadísticamente significativas.

La población a la cual se aplicará la estrategia pedagógica está constituida por la totalidad de estudiantes inscritos en la asignatura de física I de las diferentes carreras de la Escuela Tecnológica Instituto Técnico Central (ETITC) de Bogotá, Colombia, Establecimiento público de educación superior. Para el presente caso, la muestra asignada lo constituyen dos de los cursos de física I, mecánica, conformados por 21 y 18 estudiantes, quienes cumplen con regularidad su asistencia a los espacios académicos asignados. El segundo de ellos sirvió como grupo control y no se vio intervenido, mientras que al primer grupo se le aplicó la intervención pedagógica. Dado que el número total de estudiantes de física mecánica de la ETITC es de 130, asumida como el universo de la población, se considera que el tamaño de la muestra cumple con los requerimientos estadísticos.

\section{Estrategia pedagógica}

En relación con la puesta en escena, en primer lugar, se invita a los estudiantes de los cursos de física I (mecánica) designados para el desarrollo del trabajo de campo, a participar en el proyecto, haciendo una introducción al concepto de pensamiento crítico, y su importancia desde el punto de la investigación, lo que se espera de ellos durante el curso y lo que la metodología les puede aportar al desarrollo de habilidades necesarias para su desempeño personal y profesional.

El trabajo estructurado de la estrategia pedagógica consta de cinco momentos cíclicos en cada unidad: una consulta bibliográfica, enfocada al desarrollo de la habilidad de evaluación de la calidad de una fuente de información; el momento de la clase magistral tendiente a ser un dialogo socrático a partir de la consulta previa de los temas; el uso de simuladores virtuales disponibles en INTERNET, buscando motivar al estudiante al uso de herramientas diferentes a la clase magistral y que promueva su posición activa al incitarlo a la generación de conjeturas sobre las condiciones dadas por el modelo teórico y contrastarlas con lo que se observa en la realidad virtual; en cuarto término, se enfrenta al estudiante a la solución de una serie de ejercicios denominados "problemas analíticos en física", cuya característica fundamental es la de ser problemas mal definidos (Mayer, 1986) entendidos como aquellos en los que el punto de partida y el proceso de solución no es totalmente claro, además es posible que se tengan varias soluciones diferentes entre sí, pero totalmente válidas, implicando exigir al estudiante hacia el análisis y reflexión. Finalmente se tiene la "exploración conceptual", estrategia inmersa y justificada desde la metodología de "rutinas de pensamiento" (Swartz y Perkins, 1989). Esta estrategia conlleva que el estudiante genere una rutina de pensamiento para las sesiones, pretendiendo que el estudiante observe, comprenda y describa. Se busca que los cinco escenarios de la intervención pedagógica están centrados en el desarrollo de habilidades de pensamiento, objetivo central del trabajo.

La estrategia pedagógica se resguarda en las temáticas básicas del curso de física I, que corresponden al estudio del movimiento en la cinemática, las leyes de Newton y sus implicaciones en el capítulo de dinámica y el tema de trabajo y energía, aplicado a conceptos mecánicos. El rol del docente durante todo el proceso de intervención es de acompañamiento más que de transmisor de conocimientos, su papel se potencializa en el proceso de planeación y generación de las estrategias aplicadas.

\section{Evaluación del nivel de habilidades de pensamiento crítico}

Para la determinación del nivel de habilidades de pensamiento crítico en los estudiantes se indagó sobre las pruebas disponibles más relevantes por ser generadas por grupos de trabajo de amplia experiencia y con un gran rango de aplicación a nivel mundial:The California Critical Thinking Test; Prueba de pensamiento crítico de Cornell; Evaluación de pensamiento crítico de Watson - Glaser; International Critical thinking test. The Ennis - Weir Critical Thinking Essay Test y el Test PENCRISAL de la Universidad de Salamanca. Dado que la mayoría de pruebas son de selección múltiple, tipo test, es decir que son pruebas cerradas, se tienen en cuenta los estudios realizados por Saiz y Rivas (2012) quienes concluyen que; en primer lugar, una prueba cerrada permite evaluar las respuestas, más no el proceso de pensamiento seguido por el estudiante, en segundo lugar, las pruebas citadas solo exigen al estudiante la comprensión de una tarea, sin tener en cuenta la producción individual, para finalmente argumentar que la complejidad de la tarea está casi siempre alejada de la cotidianidad del estudiante, por lo que resulta poco motivante para el estudiante.

Dados los argumentos anteriores se decide usar el test PENCRISAL de la Universidad de Salamanca para los objetivos del estudio. La prueba PENCRISAL está conformada por 35 ítems, que evalúan las capacidades de deducción, inducción, razonamiento práctico, toma de decisiones y solución de problemas. Cada uno de los ítems anteriores se evalúa a través de 7 preguntas intercaladas aleatoriamente dentro de 
la prueba. Para cada ítem se tienen tres posibles puntajes: 0 si la respuesta es incorrecta; 1 punto cuando solamente la solución es correcta, pero no se argumenta adecuadamente, y 2 puntos si da la respuesta correcta, justificando el porqué de la misma. El idioma y el haber sido validada en contextos Latinoamericanos fueron también tenidos en cuenta.

A manera de ilustración, se cita una de las preguntas del test, aprobada para su publicación, dado que es un producto privado por el que se deben pagar derechos para su aplicación. "El entrenador personal de Juan le ha dicho que solo si entrena dos horas al día aprobará las pruebas del cuerpo de bomberos. Juan está preocupado porque, al no haber entrenado las dos horas requeridas, cree que no va a superar ese examen" ¿Es valida la preocupación de Juan? Justifica tu respuesta. Se puede ampliar información en el texto de Saíz y Rivas (2012).

\section{RESULTADOS}

En primer lugar, se describen las características generales de la muestra en la tabla 1. Adicionalmente se identificó la variable nivel económico, en la que la totalidad de la muestra pertenecen a los estratos 1, 2 y 3 , sin diferencias significativas entre los dos grupos. En el caso colombiano dichos estratos corresponden a personas con ingresos mínimos catalogados como de ingresos bajo-bajo, bajo y medio-bajo respectivamente. Los resultados obtenidos del curso intervenido están detallados en la tabla \# 2. En la tabla \# 3 se pueden observar los resultados obtenidos en el caso del curso tomado como control, el cual mantuvo su metodología tradicional de exposición magistral de los temas.

En seguida se procede a la determinación de si existen diferencias en el rendimiento en pensamiento crítico de los estudiantes participantes en el estudio desde dos líneas de trabajo. En primer lugar, la comparación entre los resultados pre y post test para el grupo intervenido, y posteriormente la comparación en el rendimiento en pensamiento crítico entre los resultados pre y post test para el grupo control. Para tal análisis, se ha empleado el paquete estadístico IBM SPSS Statistics. Dado el tamaño de la muestra, deben usarse pruebas no paramétricas, en el presente caso se usa el test de Wilcoxon que arrojó, para el caso de la comparación test - pos test para el grupo intervenido los resultados observados en la tabla \#4.

Tabla 1: Características generales de la muestra

\begin{tabular}{|l|c|c|c|c|c|c|c|}
\hline Grupo & $\mathrm{N}$ & Hombres \% & Mujeres \% & Media edad & d.t. & IC 95\% & Rango años \\
\hline Intervenido & 21 & 71.4 & 28.6 & 19.66 & 2.834 & $18.37-20.95$ & $17-26$ \\
\hline Control & 18 & 83.3 & 16.7 & 19.46 & 3.340 & $18.31-20.60$ & $16-36$ \\
\hline
\end{tabular}

Tabla 2: Descriptiva de variables PENCRISAL pre - post grupo intervenido

\begin{tabular}{|l|c|c|c|c|c|}
\hline \multicolumn{1}{|c|}{ Variables } & $\mathrm{N}$ & Media & Desviación & IC 95\% & Rango \\
\hline Puntaje total pre & 21 & 15.62 & 5 & $13,36-17,88$ & $3-24$ \\
\hline P. Deductivo pre & 21 & 2.33 & 1.906 & $1.47-3.20$ & $0-7$ \\
\hline P. Inductivo pre & 21 & 3.43 & 1.248 & $2.86-4.00$ & $1-5$ \\
\hline R. Practico pre & 21 & 2.1 & 1.7 & $1.32-2.87$ & $0-6$ \\
\hline Toma decisiones & 21 & 3.81 & 1.887 & $2.95-4.67$ & $0-7$ \\
\hline Sol. problemas & 21 & 3.95 & 1.717 & $3.17-4.73$ & $1-8$ \\
\hline Puntaje total post & 21 & 16.19 & 4.523 & $14.13-18.25$ & $7-25$ \\
\hline P. Deductivo post & 21 & 4.24 & 1.64 & $3.49-4.98$ & $2-7$ \\
\hline P. Inductivo post & 21 & 2.71 & 1.309 & $2.12-3.31$ & $0-5$ \\
\hline R. Práctico post & 21 & 3.24 & 1.786 & $2.43-4.05$ & $1-8$ \\
\hline Toma decisiones & 21 & 4.1 & 2.427 & $2.99-5.20$ & $0-9$ \\
\hline Sol. problemas & 21 & 3.9 & 1.729 & $3.12-4.69$ & $1-7$ \\
\hline
\end{tabular}

En lo referente a los resultados obtenidos en el puntaje total de pensamiento crítico, así como en los cinco factores evaluados para el grupo intervenido, se identifica que solo existen diferencias significativas en lo concerniente a razonamiento deductivo y razonamiento práctico, en donde el valor que arrojó Z fue de 3.038 y de 2.638 respectivamente. Tal como se podría prever, estos resultados son mayores en el post test que en el test inicial. En la tabla \# 5 se tiene el mismo análisis para el caso del grupo control, usando nuevamente la prueba no paramétrica correspondiente. 
Tabla 3: Descriptiva de variables PENCRISAL pre - post grupo control

\begin{tabular}{|l|c|c|c|c|c|}
\hline \multicolumn{1}{|c|}{ Variables } & $\mathrm{N}$ & Media & Desviación & IC 95\% & Rango \\
\hline Puntaje total pre & 18 & 20.72 & 4.51 & $16.99-22.79$ & $14-29$ \\
\hline P. Deductivo pre & 18 & 2.833 & 1.465 & $2.10-3.56$ & $0-6$ \\
\hline P. Inductivo pre & 18 & 3.389 & 1.461 & $2.66-4.11$ & $1-6$ \\
\hline R. Practico pre & 18 & 3.889 & 2.026 & $2.88-4.90$ & $2-10$ \\
\hline Toma decisiones & 18 & 4.556 & 1.199 & $3.96-5.15$ & $2-10$ \\
\hline Sol. problemas & 18 & 6.056 & 1.765 & $5.18-6.93$ & $2-9$ \\
\hline Puntaje total post & 18 & 19.89 & 5.83 & $16.98-22.79$ & $8-35$ \\
\hline P. Deductivo post & 18 & 2.722 & 1.364 & $2.04-3.40$ & $0-5$ \\
\hline P. Inductivo post & 18 & 3.500 & 1.543 & $2.73-4.27$ & $2-7$ \\
\hline R. Practico post & 18 & 2.944 & 2.127 & $1.89-4.00$ & $1-9$ \\
\hline Toma decisiones & 18 & 5.056 & 2.775 & $3.67-6.43$ & $0-11$ \\
\hline Sol. problemas & 18 & 5.667 & 1.91 & $4.72-6.62$ & $2-9$ \\
\hline
\end{tabular}

Tabla 4: Comparación de medias Test - Post test a través de Test de Wilcoxon: grupo Intervenido

\begin{tabular}{|c|c|c|c|c|c|c|}
\hline \multirow[t]{2}{*}{ Variables } & & \multirow[b]{2}{*}{$\mathrm{N}$} & Media & Desviación & \multicolumn{2}{|c|}{ Test de Wilcoxon } \\
\hline & & & & & Z & p-sig. \\
\hline \multirow{2}{*}{ Total } & Pre & 21 & 15.62 & 4.965 & \multirow{2}{*}{0.488} & \multirow{2}{*}{0.626} \\
\hline & Post & 21 & 16.19 & 4.523 & & \\
\hline \multirow{2}{*}{ P. Deductivo } & Pre & 21 & 2.33 & 1.906 & \multirow{2}{*}{3.038} & \multirow{2}{*}{0.002} \\
\hline & Post & 21 & 4,24 & 1.640 & & \\
\hline \multirow{2}{*}{ P. Inductivo } & Pre & 21 & 3.43 & 1.248 & \multirow{2}{*}{1.804} & \multirow{2}{*}{0.071} \\
\hline & Post & 21 & 2.71 & 1.309 & & \\
\hline \multirow{2}{*}{ R. Práctico } & Pre & 21 & 2.10 & 1.700 & \multirow{2}{*}{2.638} & \multirow{2}{*}{0.008} \\
\hline & Post & 21 & 3.24 & 1.786 & & \\
\hline \multirow{2}{*}{ Toma decisiones } & Pre & 21 & 3.81 & 1.887 & \multirow{2}{*}{0.477} & \multirow{2}{*}{0.634} \\
\hline & Post & 21 & 4.10 & 2.427 & & \\
\hline \multirow{2}{*}{ Solución problemas } & Pre & 21 & 3.95 & 1.717 & \multirow{2}{*}{0.045} & \multirow{2}{*}{0.964} \\
\hline & Post & 21 & 3.90 & 1.729 & & \\
\hline
\end{tabular}

Tabla 5: Comparación de medias Test - Post test a través de Test de Wilcoxon: grupo control

\begin{tabular}{|c|c|c|c|c|c|c|}
\hline \multirow{2}{*}{ Variables } & & \multirow{2}{*}{$\mathrm{N}$} & \multirow[t]{2}{*}{ Media } & \multirow[t]{2}{*}{ Desviación } & \multicolumn{2}{|c|}{ Test de Wilcoxon } \\
\hline & & & & & Z & $p$ - sig. \\
\hline \multirow{2}{*}{ Total } & Pre & 18 & 20.72 & 4.510 & \multirow{2}{*}{0.687} & \multirow{2}{*}{0.492} \\
\hline & Post & 18 & 19.89 & 5.830 & & \\
\hline \multirow{2}{*}{ P. Deductivo } & Pre & 18 & 2.833 & 1.465 & \multirow{2}{*}{0.141} & \multirow{2}{*}{0.888} \\
\hline & Post & 18 & 2.722 & 1.364 & & \\
\hline \multirow{2}{*}{ P. Inductivo } & Pre & 18 & 3.389 & 1.461 & \multirow{2}{*}{0.029} & \multirow{2}{*}{0.977} \\
\hline & Post & 18 & 3.500 & 1.543 & & \\
\hline \multirow{2}{*}{ R. Práctico } & Pre & 18 & 3.889 & 2.026 & \multirow{2}{*}{1.599} & \multirow{2}{*}{0.110} \\
\hline & Post & 18 & 2.944 & 2.127 & & \\
\hline \multirow{2}{*}{ Toma decisiones } & Pre & 18 & 4.556 & 1.199 & \multirow{2}{*}{0.599} & \multirow{2}{*}{0.549} \\
\hline & Post & 18 & 5.056 & 2.775 & & \\
\hline \multirow{2}{*}{ Solución problemas } & Pre & 18 & 6.056 & 1.765 & \multirow{2}{*}{0.572} & \multirow{2}{*}{0.567} \\
\hline & Post & 18 & 5.667 & 1.910 & & \\
\hline
\end{tabular}


Para el caso del grupo control, el Test de Wilcoxon no predice diferencias significativas entre las medias de los datos totales del pre test y el post test, ni en ninguno de los ítems que evalúa el test, la mayor variación corresponde al razonamiento práctico con un valor de $Z$ de 1.599, sin embargo, no supera el valor crítico de 1.96 dado por el Test de Wilcoxon, por otro lado, la variación fue hacia abajo, es decir el puntaje disminuye para este caso. De aquí se concluye que, para el caso de una instrucción clásica magistral, no se detecta ninguna variación significativa en las habilidades de pensamiento crítico evaluadas en los estudiantes del grupo control, de hecho en general los puntajes pos test son menores que los puntajes pre test para este grupo, exceptuando el caso de toma de decisiones cuya media paso de 4.556 a 5.056.

En lo referente a los resultados generales de las habilidades de pensamiento crítico evaluadas, se encuentra un rendimiento muy bajo, dado que solo se obtiene un valor máximo en las medias de 20.72 , comparada con el valor máximo posible de 70 , los resultados son preocupantes, Se intuye que los estudiantes no tienen una formación que desarrolle en ellos habilidades de pensamiento crítico. A modo de comparación si se comparan los resultados obtenidos por el grupo intervenido con el baremo de la puntuación obtenida por el grupo peruano al ser evaluado con la misma prueba, dichas puntuaciones únicamente se encuentran entre los centiles 12 y 30 de la prueba peruana (Rivas et al., 2014)

En lo que respecta a la hipótesis de trabajo, de que es posible generar una intervención pedagógica que promueva el desarrollo de habilidades de pensamiento crítico en los estudiantes a través del área disciplinar de la física, se puede generar una esperanza en que sea de esa manera. Se observa una diferencia estadísticamente significativa en sentido positivo en dos de las cinco habilidades evaluadas, particularmente en los puntajes de pensamiento deductivo y razonamiento práctico para el caso del curso intervenido, mientras que este mismo hecho no se observó en las habilidades medidas en el curso tomado como control. Dado el control de las variables intervinientes en el estudio, tal variación puede atribuirse a la estrategia pedagógica. Podemos concluir que es posible que la estrategia pedagógica diseñada haya generado un aumento en dichas habilidades, sin embargo, deben anotarse ciertos aspectos.

En primer lugar, el tamaño de la muestra no es lo suficientemente grande como para sacar conclusiones definitivas. Se propone continuar el estudio ampliando a una población más amplia de la institución o en otras instituciones similares. En segundo lugar, el periodo sobre el cual se aplicó la intervención pedagógica fue de un semestre, tiempo mínimo para observar un cambio realmente permanente y duradero. Sin embargo, es este mismo aspecto el que podría generar más expectativas, dado que con una mayor exposición a la intervención pedagógica los efectos podrían ser más notorios que los detectados en el trabajo actual. Se propone generar un proyecto que implique la aplicación permanente de una intervención pedagógica encaminada al desarrollo de habilidades de pensamiento.

Dado que la prueba PENCRISAL aplica preguntas abiertas en un contexto de situaciones cotidianas, los resultados hacen prever que es posible aportar, aún desde la enseñanza de las ciencias básicas al desarrollo integral del individuo, generando habilidades transversales que no solo impliquen el avance en la capacidad de resolver problemas de la ciencia y la técnica, sino, aún más importante, problemas de su entorno cotidiano, de familia, de trabajo en equipo, de sociedad.

De acuerdo con los resultados se puede esperar que sea posible y necesario diseñar propuestas pedagógicas basadas en los sustentos teórico - prácticos de investigadores reconocidos como David Perkins, Edward de Bono, Rafi Feuerstein, Mattew Lipman, entre otros, que generen habilidades de pensamiento crítico en los estudiantes, lo cual a su vez redundará en el progreso de las sociedades Latinoamericanas.

\section{CONCLUSIONES}

De acuerdo a los resultados obtenidos a través de los diferentes análisis estadísticos se puede concluir lo siguiente.

La diferencia estadísticamente significativa en sentido positivo en dos de las cinco habilidades evaluadas demuestra que la intervención pedagógica conlleva un efecto en el curso intervenido.

Dado el control de las variables dicho cambio puede atribuirse a la estrategia pedagógica.

Es posible aportar desde la enseñanza de las ciencias básicas a la formación integral del individuo.

Los resultados invitan a diseñar estrategias pedagógicas basadas en la enseñanza del pensamiento crítico como aporte a la educación en Latinoamérica. 


\section{REFERENCIAS}

Aznar, I., Cáceres, M. Hinojo, M. La adquisición de competencias específicas en la Educación Superior. Evaluando la formación del psicopedagogo en la Universidad de Granada. ENSAYOS, Revista de la Facultad de Educación de Albacete. ISSN: 0214-4824 (En Línea),26. 71 - 93. (2011). https://dialnet.unirioja.es/descarga/articulo/4010568.pdf. Acceso: junio 3 (2015)

Boisvert, J. La formación del pensamiento crítico. México: Fondo de cultura económica. (2004)

Benegas, J. y Villegas, M. Influencia del Texto y del Contexto en la Resolución de Problemas de Física. LAJPE, 5(1). 217-224. (2011)

Beltran, J. y Torres, N. Caracterización de habilidades de pensamiento crítico en estudiantes de educación media a través del test HCTAES. Zona Próxima, 11, 66-85. (2009)

Betancourth, S. Desarrollo de Pensamiento Crítico en Docentes Universitarios. Una Mirada Cualitativa. Revista virtual Universidad Católica del Norte, 44, 238 - 252. (2015)

Browne, M. y Keeley, S. Asking the Right Questions: a Guide to Critical Thinking. Estados Unidos: Pearson. (2015)

Castiblanco, O. Y Vizcaino, D. Pensamiento Crítico y Reflexivo Desde la Enseñanza de la Física. Revista Colombiana de Física. 38(2), 674 - 677. (2006)

Colás, P. Buendía L. y Hernández F. Competencias Científicas para la Elaboración de una Tesis Doctoral. Barcelona: Editorial Davinci. (2009)

Dewey, J. Cómo Pensamos. Madrid, España: Paidós. (2007)

Ennis R. An outline of goals for a Critical Thinking curriculum and its assessment. (En línea). University of Illinois (2000). http://www.criticalthinking.net/goals.html_Acceso: Octubre 18 (2015)

Franco, A. Almeida, y L. Saiz, C. Pensamiento crítico: reflexión sobre su lugar en la Enseñanza Superior. Educatio Siglo XXI. 32(2). 81-96. (2014)

Gangoso, Z. Investigaciones en Resolución de Problemas en Ciencias. Investigación en Enseñanza de las Ciencias. 4(1). 7 - 50. (1999)

Girelli, M. Dima, G. Reinoso, S. y Baumann, L. Habilidades de Pensamiento Crítico y Superior Desarrolladas por un Grupo de Alumnos de Carreras de Física Universitaria. Resultados de Entrevistas Realizadas a sus Docentes. Latin - American Journal of Physics Education. 4(1). 194 - 199. (2010)

Inzunza, J. y Brincones, I. Aprendizaje de la Física por Resolución de Problemas: Caso de Estudio en Alcala de Henares. Theoria. 19(2). 51-59. (2010)

Lara-Barragán, A. y Cerpa, G. Enseñanza de la Física y Desarrollo del Pensamiento Crítico. Latin-American Journal of Physics Education. 8(1). 52 - 59. (2014)

Lipman, M. Pensamiento Complejo y Educación. Madrid: Ediciones de la Torre. (2001)

Mayer, R. Psicología de la Educación. Madrid: Prentice Hall. (2002)

Maclure, S. y Davies, P. Aprender a Pensar, Pensar en Aprender. Barcelona: Gedisa Editores. (2003)

Olivares. S. y Heredia. Y. Desarrollo del pensamiento Crítico en ambientes de aprendizaje basado en problemas en estudiantes de educación superior. Revista Mexicana de Investigación Educativa. 17(54). 759-778. (2012)

Ozman, S. Mokhtar, M. Abu, M. y Mohammad, S. Pertinent Elements of Critical Thinking and Mathematical Thinking Used by Practicing Civil Engineers. International Journal of Sciences (En línea). 23 (2). 381 - 395. (2015). gssrr.org/index.php?journal=JournalOfBasicAndApplied. Acceso: Febrero 04 (2016)

Perkins, D. La Escuela Inteligente. Barcelona: Gedisa Editorial. (2008)

Polya, G. Cómo plantear y resolver problemas. México: Trillas. (1965)

Pozo, J. I. La solución de problemas. Madrid: Santillana. (1999)

Rivas, S. Morales, P. Saíz, C. Propiedades psicométricas de la adaptación peruana de la prueba de pensamiento crítico PENCRISAL . Avaliação Psicológica. 13(2). 257-268. (2014)

Saíz, C. Y Rivas, S. Validación y propiedades psicométricas de la prueba de pensamiento crítico PENCRISAL. Revista Electrónica de Metodología Aplicada. 17 (1). 18 - 34. (2012)

Swartz, R. y Perkins, D. Teaching Thinking: Issues and Approaches. Boston: Midwest Publications. (1989) 